\title{
Molding of Three-Dimensional Microstructures of Gels
}

\author{
Min D. Tang, Andrew P. Golden, and Joe Tien* \\ Department of Biomedical Engineering, Boston University, 44 Cummington Street, Boston, Massachusetts 02215
}

Received July 31, 2003; E-mail: jtien@bu.edu

This Communication describes the use of patterned elastomeric stamps to fabricate three-dimensional (3D) microstructures of hydrogels. Hydrogels are indispensable for many chemical and biological applications, including electrophoretic separation, chromatography, drug delivery, biosensing, and tissue engineering. ${ }^{1}$ The ability to pattern the topologies of gels at the microscale is desirable in these applications, because microscale structures are often more sensitive and versatile than their macroscale counterparts are. To date, patterning of gels at the microscale has relied primarily on photopolymerization of liquid precursors; $;, 3$ this strategy cannot be used with many types of gels, such as those made of proteins or sugars. Here, we introduce a general strategy for microfabrication of gels: the use of poly(dimethylsiloxane) (PDMS) stamps to mold, release, and stack gels into 3D structures, and the use of surface modification to promote the release or adhesion of molded gels to a substrate.

Our work builds upon the studies of Whitesides and others in soft lithography, which use patterned PDMS stamps to generate microscale structures in photoresist, polymers, and metals. ${ }^{4,5} \mathrm{We}$ first identified surface treatments for PDMS that allowed release of a molded gel from a stamp, because gels that were molded against untreated PDMS often adhered to the stamps and deformed irreversibly upon separation from them. Of the surface treatments that we tested, only modification of PDMS stamps by hexa(ethylene glycol)-terminated self-assembled monolayers (SAMs), or by adsorbed monolayers of bovine serum albumin (BSA), allowed distortion-free separation of stamps from gels molded against them. ${ }^{6}$ These treatments greatly reduce nonspecific adsorption of protein; ${ }^{7}$ we suspect that their ability to promote release of gels results from a decrease in adsorption of soluble gel precursors onto treated stamps.

Modification of PDMS stamps with these treatments allowed the application of standard soft lithographic techniques, ${ }^{8}$ such as replica molding, microtransfer molding $(\mu \mathrm{TM})$, and micromolding in capillaries (MIMIC), to the microfabrication of gels. ${ }^{9}$ Figure 1 shows images of microstructures molded in collagen, using modified PDMS stamps; we obtained similar results when molding other protein-based gels, such as gelatin and the tumor extract Matrigel, as well as sugar-based gels such as agarose. Replica molding, $\mu \mathrm{TM}$, or MIMIC of liquid precursors generated monolithic gels (with surface relief), isolated microstructures, or interconnected networks, respectively, with a spatial resolution of $\leq 5 \mu \mathrm{m}$ (Figure 1A, inset). Microtransfer molding against substrates that promoted or resisted the adhesion of gels generated supported arrays of collagen gels and a suspension of free-standing gels, respectively (Figure 1B). These gels were dimensionally stable for several days in saline at $4{ }^{\circ} \mathrm{C}$.

Many potential applications of microstructured gels require the development of structures that are more complex than the uniform gels shown in Figure 1. For instance, the ability to generate multilayered gels that contain living cells may enable the engineering of spatially complex biological tissues..$^{10}$ Likewise, the ability

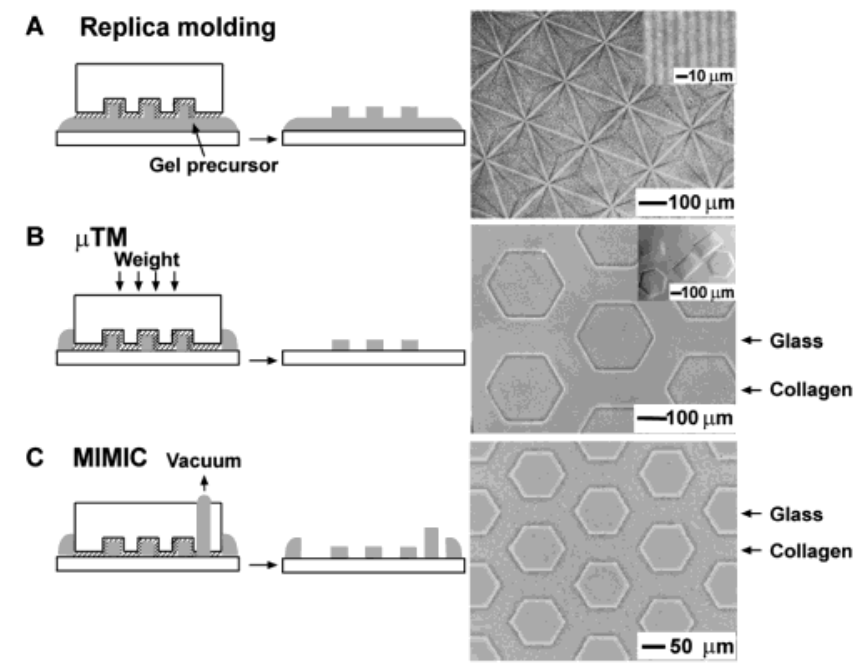

Figure 1. Schematic diagram of the micromolding of hydrogels against a modified ${ }^{6}$ PDMS stamp, and the resulting phase-contrast optical micrographs of molded collagen gels. The shaded regions represent gels and their liquid precursors. (A) Replica molding: Connected, upright pyramids (100 $\mu \mathrm{m}$ on a side, $100 \mu \mathrm{m}$ peak-to-base) on a glass coverslip. (Inset) Lines (5 $\mu \mathrm{m}$ wide, $5 \mu \mathrm{m}$ thick, $5 \mu \mathrm{m}$ spaced) (B) $\mu \mathrm{TM}$ : An array of hexagons (100 $\mu \mathrm{m}$ on a side, $100 \mu \mathrm{m}$ thick) on a glass coverslip. (Inset) Hexagonal free-standing gels that detached from a coverslip coated by an adsorbed layer of BSA. (C) MIMIC: A hexagonal mesh $(50 \mu \mathrm{m}$ on a side, $50 \mu \mathrm{m}$ thick $)$ on a glass coverslip. In each image, scattering from collagen fibers contributes to the roughness of the regions where gels are present.

to control the $3 \mathrm{D}$ arrangement of cells in gels would provide new tools for biological research. ${ }^{11}$ Thus, we have developed methods to fabricate 3D composites from gels (Figure 2). These composites consisted of (1) patterned arrays of cells in gels, (2) coplanar arrays of distinct populations of cells, and (3) stacked layers of gels. In each case, appropriate surface treatments enabled molded gels to adhere or detach selectively from substrates.

We used $\mu \mathrm{TM}$ or MIMIC to generate isolated (Figure 2A, left) and interconnected (Figure 2B, left) microstructures of collagen gels, respectively, in which human fibroblasts ${ }^{12}$ were suspended $\left(\sim 10^{9}\right.$ cells $\left./ \mathrm{mL}\right)$. We subsequently used these arrays of gels to generate a coplanar composite, by placing a droplet $(\sim 5 \mu \mathrm{L})$ of a suspension of cells in liquid collagen precursors at a corner of the array, and by sandwiching the droplet between a BSA-coated glass coverslip and the array (Figure 2A). The liquid suspension filled the space between the gelled collagen structures by capillary force and was confined to the same height as the array by the glass coverslip. Gelation of this suspension resulted in a coplanar composite of an array of isolated gels and an interconnected mesh, both of which contained separate populations of cells ${ }^{13}$ (illustrated in Figure 2A by prelabeling the cells with red and green fluorescent dyes $^{14}$ ).

To generate a bilayered collagen gel that was embedded with cells, we stacked a patterned layer of gel (at a relative angle of $\sim 30^{\circ}$ ) onto another patterned layer that was molded onto a substrate 
A
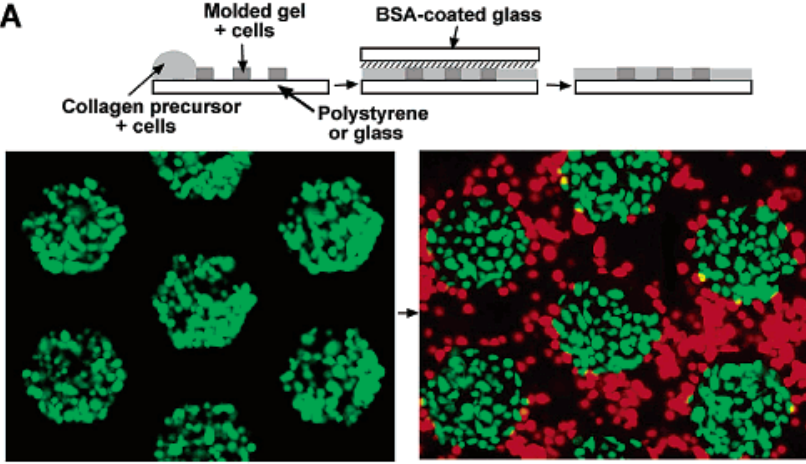

B
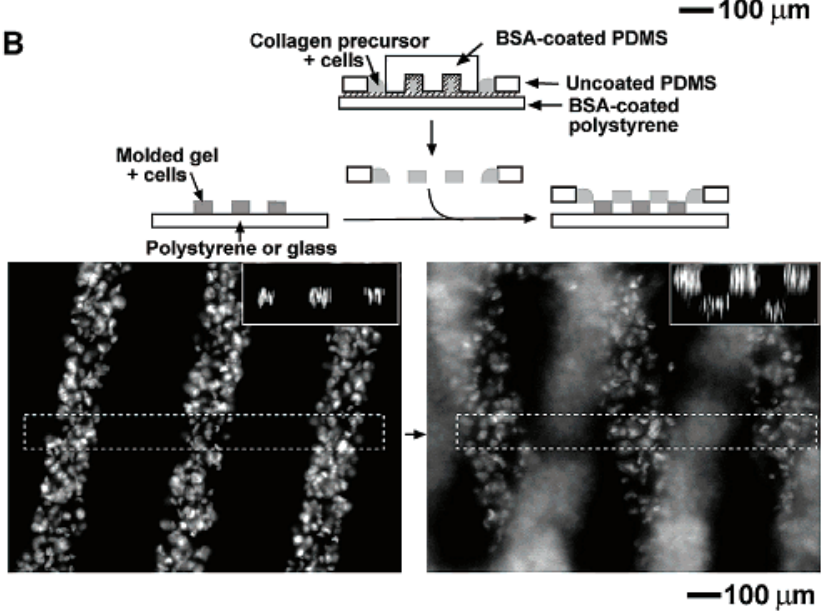

Figure 2. Schematic diagrams of the microfabrication of composite gels, and fluorescence images of cells in these gels. The shaded regions represent collagen gels in which fibroblasts are embedded. (A) (Left) An array of hexagonal gels (100 $\mu \mathrm{m}$ on a side, $100 \mu \mathrm{m}$ thick) and cells made by $\mu \mathrm{TM}$ on a glass coverslip. (Right) A composite structure that consists of an array of isolated hexagonal gels and a separate gel filling the spaces between the isolated gels on a glass coverslip. ${ }^{13}$ Fibroblasts in and between the hexagonal gels were prelabeled with green and red fluorescent dyes, respectively. ${ }^{14}$ (B) (Left) Lines (100 $\mu \mathrm{m}$ wide, $100 \mu \mathrm{m}$ thick) of gel and cells formed by MIMIC on a glass coverslip. Nuclei of fibroblasts were labeled with the fluorescent dye Hoechst $33342(1 \mu \mathrm{g} / \mathrm{mL}$; Molecular Probes). (Right) A bilayered structure that is composed of two layers of lines at a relative angle of $\sim 30^{\circ}$ on a glass coverslip. The top layer is in focus, and the layer below is out-of-focus. (Insets) Cross-sectional views (corresponding to the regions outlined by dashed boxes) of deconvolved images. ${ }^{15}$

(Figure 2B). Because the fragility of gels precludes the use of tweezers to manually manipulate and stack layers, we used a frame of PDMS to transport the top layer of gel. This top layer was formed by molding a suspension of cells in liquid collagen precursors against a PDMS stamp and a substrate (both treated to allow release of the gel), and the PDMS frame (untreated); once gelled, the molded suspension selectively adhered to the frame. We placed the frame, with attached gel in saline, on top of a substrate on which we had molded a layer of gels with embedded cells. Gentle removal of the surrounding solution brought the two gel microstructures into irreversible contact to form a bilayer. To demonstrate the relative spatial orientation of the two layers, we labeled the nuclei of the cells with a fluorescent dye and reconstructed cross sections of the sample by constrained iterative deconvolution ${ }^{15}$ of a stack of fluorescence images. In these sections, nuclei in the gels formed adjacent rectangular groups; this arrangement suggests that the layers came into direct contact while maintaining their spatial definition (Figure 2B, right, inset). Thus, we have demonstrated methods to construct 3D composites of gels, in a single plane or as a stack of two layers; we believe that, just as in the fabrication of 3D microfluidic devices, ${ }^{4,16}$ the repeated application of overlaying and stacking has the potential to generate multilayered 3D composites with complex topologies in gels. Cells in these gels remained viable and were able to spread in the gels within $\sim 1 \mathrm{~d}$. These microstructures enable control over the initial arrangement of cells and may be used for the study of remodeling and cell migration in $3 \mathrm{D}$.

Using treated PDMS to mold, overlay, and stack individual layers represents a general strategy for $3 \mathrm{D}$ microfabrication of gels and is broadly applicable to thermosetting, polymerizable, or ionsensitive hydrogels. Our results suggest that surfaces that resist nonspecific adsorption of protein readily allow detachment of molded hydrogels. Conversely, surfaces that do not resist nonspecific adsorption promote adhesion of gels, most likely via an adsorbed monolayer. Extension of our results to the microfabrication of nonaqueous organogels ${ }^{17,18}$ may require the use of stamps that resist nonspecific adsorption of polymers from organic solutions.

Acknowledgment. This work was supported by the Whitaker Foundation (RG-02-0344) and a Boston University Provost's Innovation Award. M.T. was supported by the BU Women's Council. We thank George Whitesides and Christopher Chen for materials, and Celeste Nelson, Doug Fishkind, and Mark Schomer for interesting discussions.

\section{References}

(1) Li, Y.; Tanaka, T. Annu. Rev. Mater. Sci. 1992, 22, 243-277.

(2) Nguyen, K. T.; West, J. L. Biomaterials 2002, 23, 4307-4314

(3) Beebe, D. J.; Moore, J. S.; Bauer, J. M.; Yu, Q.; Liu, R. H.; Devadoss, C.: Jo, B. H. Nature 2000, 404, 588-590.

(4) Whitesides, G. M.; Ostuni, E.; Takayama, S.; Jiang, X.; Ingber, D. E. Annu. Rev. Biomed. Eng. 2001, 3, 335-373.

(5) Unger, M. A.; Chou, H. P.; Thorsen, T.; Scherer, A.; Quake, S. R. Science 2000, 288, 113-116.

(6) PDMS stamps were modified either by (1) evaporation of gold (15 nm, with $1.5 \mathrm{~nm}$ of titanium as adhesion promoter) on the stamp, followed by immersion of the stamp in a $0.1 \mathrm{mM}$ ethanolic solution of an alkanethiol or (2) adsorption of a monolayer of protein $(10-10000 \mu \mathrm{g} / \mathrm{mL}$ in phosphate-buffered saline) onto the stamp. (a) Surfaces that resisted adhesion of gels were $\mathrm{SAM}$ of $\mathrm{HS}\left(\mathrm{CH}_{2}\right)_{11}\left(\mathrm{OCH}_{2} \mathrm{CH}_{2}\right)_{6} \mathrm{OH}$ on $\mathrm{Au}$; adsorbed BSA. (b) Surfaces that promoted adhesion of gels were SAMs of HS$\left(\mathrm{CH}_{2}\right)_{15} \mathrm{CH}_{3}, \mathrm{HS}\left(\mathrm{CH}_{2}\right)_{11} \mathrm{OH}$, or $\mathrm{HS}\left(\mathrm{CH}_{2}\right)_{11} \mathrm{CO}_{2} \mathrm{H}$ on $\mathrm{Au}$; glass (exposing - $\mathrm{SiOH}$ groups); polystyrene.

(7) Ostuni, E.; Chapman, R. G.; Liang, M. N.; Meluleni, G.; Pier, G.; Ingber, D. E.; Whitesides, G. M. Langmuir 2001, 17, 6336-6343.

(8) Xia, Y. N.; Whitesides, G. M. Аnnu. Rev. Mater. Sci. 1998, 28, 153184 .

(9) Liquid, acidic collagen precursor (BD Biosciences) was gelled by neutralization with $\mathrm{NaOH}$ and incubation at $37^{\circ} \mathrm{C}$ for $1 \mathrm{~h}$

(10) Weinberg, C. B.; Bell, E. Science 1986, 231, 397-400.

(11) Grinnell, F. Trends Cell Biol. 2003, 13, 264-269.

(12) Normal human dermal fibroblasts (BioWhittaker) were routinely cultured at $37{ }^{\circ} \mathrm{C}, 5 \% \mathrm{CO}_{2}$, and $100 \%$ humidity in Dulbecco's modified Eagle's medium that contained $10 \%$ calf serum (GIBCO) and $1 \%$ penicillinstreptomycin-glutamine (Invitrogen). Cells were trypsinized, washed with saline, concentrated by centrifugation ( $300 \mathrm{~g}, 2 \mathrm{~min})$, and mixed with liquid collagen precursor at $\sim 1: 1$ volume ratio.

(13) We believe that the nonuniformity in cell distribution in Figure 2A arise from inadequate mixing of the cell suspension with saline surrounding the molded hexagonal gels.

(14) Fibroblasts were labeled by incubation in a $1 \mu \mathrm{M}$ solution of CellTracker Orange or Green (Molecular Probes) in serum-free media at $37^{\circ} \mathrm{C}$ for 30 $\mathrm{min}$. Cells were then washed with saline three times and incubated in normal growth media for another $30 \mathrm{~min}$ before use.

(15) We obtained a Z-stack of 100-200 images with a spacing of $4.65 \mu \mathrm{m}$ per image and used a constrained iterative algorithm (AxioVision 3.1, Carl Zeiss) to reconstruct the image in 3D

(16) Anderson, J. R.; Chiu, D. T.; Jackman, R. J.; Cherniavskaya, O. McDonald, J. C.; Wu, H. K.; Whitesides, S. H.; Whitesides, G. M. Anal. Chem. 2000, 72, 3158-3164.

(17) Abdallah, D. J.; Weiss, R. G. Adv. Mater. 2000, 12, 1237-1247.

(18) Xing, B. A.; Choi, M. F.; Zhou, Z. Y.; Xu, B. Langmuir 2002, 18 , 9654-9658.

JA037677H 